Ciência e Natura, Santa Maria v.38 n.1, 2016, Jan.- Abr. p. 326 - 340

Revista do Centro de Ciências Naturais e Exatas - UFSM

ISSN impressa: 0100-8307 ISSN on-line: 2179-460X

\title{
Análise mensal, sazonal e interanual da Evapotranspiração potencial para o leste do estado do Acre, Brasil.
}

\author{
Helder José Farias da Silva ${ }^{1}$, Paulo Sergio Lucio ${ }^{1,2}$, Irving Foster Brow ${ }^{3}$ \\ ${ }^{1}$ Mestrando do Programa de Pós-Graduação em Ciências Climáticas, UFRN, Natal - RN, Brasil. \\ helderlagoia@gmail.com \\ ${ }^{2}$ Departamento de Estatística, Programa de Pós-Graduação em Ciências Climáticas, UFRN, Natal-RN, Brasil. \\ pslucio@ccet.ufrn.br \\ ${ }^{3}$ Woods Hole Research Center, Universidade Federal do Acre, Rio Branco-AC, Brasil. \\ fbrown@uol.com.br
}

\begin{abstract}
Resumo
O objetivo deste trabalho foi de investigar aspectos climatológicos (caracterização) sazonais na Evapotranspiração potencial para o leste do estado do Acre, tendo como unidade de estudo o município de Rio Branco, capital do Estado, considerando um período de 34 anos: 1980 a 2013, a partir de dados mensais de uma estação meteorológica de superfície convencional. Como metodologia adotou-se realizar, primeiramente, a consistência dos dados meteorológicos desta forma foi feito o preenchimento de falhas através da imputação de dados faltosos na série temporal por meio do Multivariate Time Series Data Imputation e testes estatísticos de homogeneidade dos dados (Run teste ou de seqüência) sugerido pela Organização Meteorológica Mundial por meio de sua nota técnica nº 81 . O método indireto de Penmann-Montheith foi utilizado para o cálculo da Eevapotranspiração potencial. Os resultados deste trabalho, mediante análise da estatística descritiva, permitiram concluir que a média anual da ETp é de 3,54 $\mathrm{mm} \mathrm{d}^{-1}$, com uma sazonalidade bastante marcante apresentando um mínimo em junho de 2,93 $\mathrm{mm} \mathrm{d}^{-1}$ e um máximo no mês de outubro de 4,13 $\mathrm{mm} \mathrm{d}^{-1}$ com um acumulado médio anual de 1290,99 mm e Desvio Padrão de $53,41 \mathrm{~mm}$ e que os meses de junho e agosto são o que apresentam maior variabilidade e o intervalo de outubro a fevereiro o de menor variabilidade.
\end{abstract}

Palavras-chave: Variabilidade climática. Penman-Monteith-FAO. Teste de Homogeneidade.

\begin{abstract}
The aim of this study was to investigate climatological aspects (characterization) in seasonal potential evapotranspiration east of Acre, with the unit of study is the city of Rio Branco, capital of the State, considering a period of 34 years from 1980 to 2013, from monthly data from a weather station in a conventional surface. The methodology was adopted to realize the consistency of meteorological data in this way, first, was made the gap filling through the imputation of missing data in time series by means of Multivariate Time Series Data imputation and statistical tests of homogeneity of data (test or Run sequence) suggested by the World Meteorological Organization through its technical note 81 the indirect method Penmann-Montheith was used to calculate the potential Evapotranspiração. The results of this work through examination of the descriptive statistics showed that the average annual ETp is $3.54 \mathrm{~mm} \mathrm{d-1}$, with a very marked seasonality in June featuring a minimum of $2.93 \mathrm{~mm} \mathrm{~d}^{-1}$ and a maximum in October from $4.13 \mathrm{~mm} \mathrm{~d}^{-1}$ with an average annual accumulated $1290.99 \mathrm{~mm}$ and Standard Deviation of $53.41 \mathrm{~mm}$ and the months of June and August are what have higher variability and the interval from October to February the lowest variability.
\end{abstract}

Keywords: Climate variability. Penman-Monteith-FAO. Homogeneity Test. 


\section{Introdução}

Em solos com cobertura vegetal e corpos d'água (rios, lagos, reservatórios de água) os processos de Evapotranspiração (ET) e Evaporação (E), respectivamente, consomem considerável quantidade de energia para o transporte vertical de vapor. Por ser a água total perdida pelo sistema, a ET deve ser determinada com maior rigor possível, a fim de ser reposta e manter sempre o sistema em condições de máximo relacionamento com o meio. Esses fluxos não podem ser negligenciados em inúmeras atividades humanas; e assim, as suas estimativas e monitoramento são extremamente importantes, principalmente no gerenciamento e planejamento dos recursos hídricos e atividades agrícolas que demandam grandes quantidades de água.

Carvalho et al., (2011), considera que a Evapotranspiração de referencia (ETo), originalmente introduzida sob o termo Evapotranspiração potencial (ETp), é um importante parâmetro agrometerológico, mas, também, é considerada elemento climático de demanda hídrica, dai sua aplicação em estudos meteorológicos, climatológicos e hidrológicos. Estes autores reforçam que o método indireto de Penman-Monteith-FAO (PM-FAO) é considerado, internacionalmente, o mais apropriado para a estimativa da ETp, sendo adotado como padrão e que já vem sendo usado há duas décadas. Este método, além de procurar representar, de maneira consistente, o fenômeno biofísico da evapotranspiração, é alimentado por quase todos os elementos meteorológicos observados em estações meteorológicas de superfície. Porém, quando há impossibilidade da aplicação deste método, é necessário averiguar qual o método mais indicado, diante das condições locais.

Carvalho et al. (2011) ressaltam que, apesar da proposição da $\mathrm{FAO}$, ainda é bastante comum o uso dos termos ETp e ETo. Os autores consideraram que para estudos climatológicos o termo ETp continue sendo o mais utilizado, pois, quase sempre, está ligado à analise do potencial hídrico de uma região, enquanto a ETo é bastante adequada para projetos e manejo de irrigação. No presente trabalho, o termo utilizado será o de ETp, pois se trata da caracterização climática do potencial hídrico de uma região. No entanto, o termo ET será usado sempre que houver referência indiscriminada ao tipo de fenômeno em discussão.

Para caracterizar a Evapotranspiração de uma localidade, é necessário dispor de uma série temporal com as observações consistentes e contínuas. No Brasil, os valores médios globais de ET encontrados para o bioma Amazônico é de 4,0 mm. dia ${ }^{-1}$ (MARQUES et al. 1980; SHUTTLEWORTH et al. 1987). Estudos têm enfatizado que as mudanças climáticas podem afetar as tendências de variáveis meteorológicas, assim como discussões sobre meio ambiente e mudanças climáticas estão tomando cada vez mais importância no cenário mundial.

Os biomas tropicais brasileiros, representado principalmente pela Floresta Amazônica, têm sido foco de atenção mundial devido a sua grande diversidade biológica e também pelos efeitos que o desmatamento, o uso e ocupação da terra em grande escala podem estar provocando no clima, na hidrologia e nos ciclos biogeoquímicos em escalas regionais e globais. No Amazônia, na porção oeste, tendências de diminuição na precipitação foram observadas em estações com mais de 15 anos de dados, mas estes resultados podem estar associados ao desmatamento rápido ocorrido nas últimas décadas (PAIVA \& CLAKE, 1995). Saatchi et al. (2013) em seu estudo sobre os efeitos de seca severa sobre a Floresta Amazônica, notaram que a área de estudo (porção ocidental da Floresta Amazônica) sofreu um forte déficit hídrico durante a estação seca de 2005 e uma perda correspondente na umidade e no dossel das árvores e que apesar da recuperação gradual das chuvas nos anos seguintes, a diminuição da copa das arvores persistiram até a próxima grande seca em 2010. Estas secas foram acompanhadas por incêndios florestais que afetaram dezenas até centenas de milhares de hectares de floresta (BROWN et al. 2011).

Poucos são as pesquisas direcionadas para o estado do Acre no que concerne a caracterização sazonal da ETp. Os estudos encontrados na literatura, no entanto, têm sido realizados com enfoque mais técnico, isto é, com o objetivo de avaliar desempenhos de métodos mais simples (que contenham o menor número de parâmetros), para estimativa da ETp, por 
meio de comparações com o método padrão PMFAO. Neste sentido, Ferraz (2008), Souza (2009), Silva et al. (2013a) e Silva et al. (2013b), utilizando dados de estações convencionais e automáticas, realizaram estudos visando verificar qual o melhor método que poderia ser utilizado na ausência de dados para implementação do método padrão para a região leste do Estado. No entanto, não existem estudos na região, que tenham explorado e analisado em profundidade os aspectos climatológicos e estatísticos da ETp de forma mais metódica.

Com o intuito de suprir a lacuna, portanto, este estudo tem como objetivo investigar aspectos sazonais e estatísticos da ETp para o município de Rio Branco (AC), leste do Acre, por um período de 34 anos (1980 a 2013) utilizando-se dados meteorológicos de superfície. Espera-se que os resultados aqui apresentados possam contribuir para a discussão (científica e política) sobre a variabilidade temporal da ET no Brasil.

\section{Procedimentos}

Caracterização climática da área de estudo.

A área de estudo corresponde ao município de Rio Branco, capital do estado do Acre (AC), que ocupa uma área de 9.222,58 km² com 336.038 habitantes, sendo $38,03 \mathrm{hb} / \mathrm{km}^{2}$, situado na porção leste do Estado, entre as

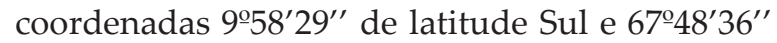
de longitude Oeste e altitude de 159 m (Figura 1) (IBGE, 2010).

A climatologia local, considerando o período dos últimos 34 anos (1980 a 2013) para Rio Branco - AC, é caracterizado por média da temperatura máxima de $31,5^{\circ} \mathrm{C}$ e as das mínimas $20,5^{\circ} \mathrm{C}$. A temperatura média compensada varia entre $23,5{ }^{\circ} \mathrm{C}$ em julho a $26,0{ }^{\circ} \mathrm{C}$ em outubro, com média anual de $25,1^{\circ} \mathrm{C}$.

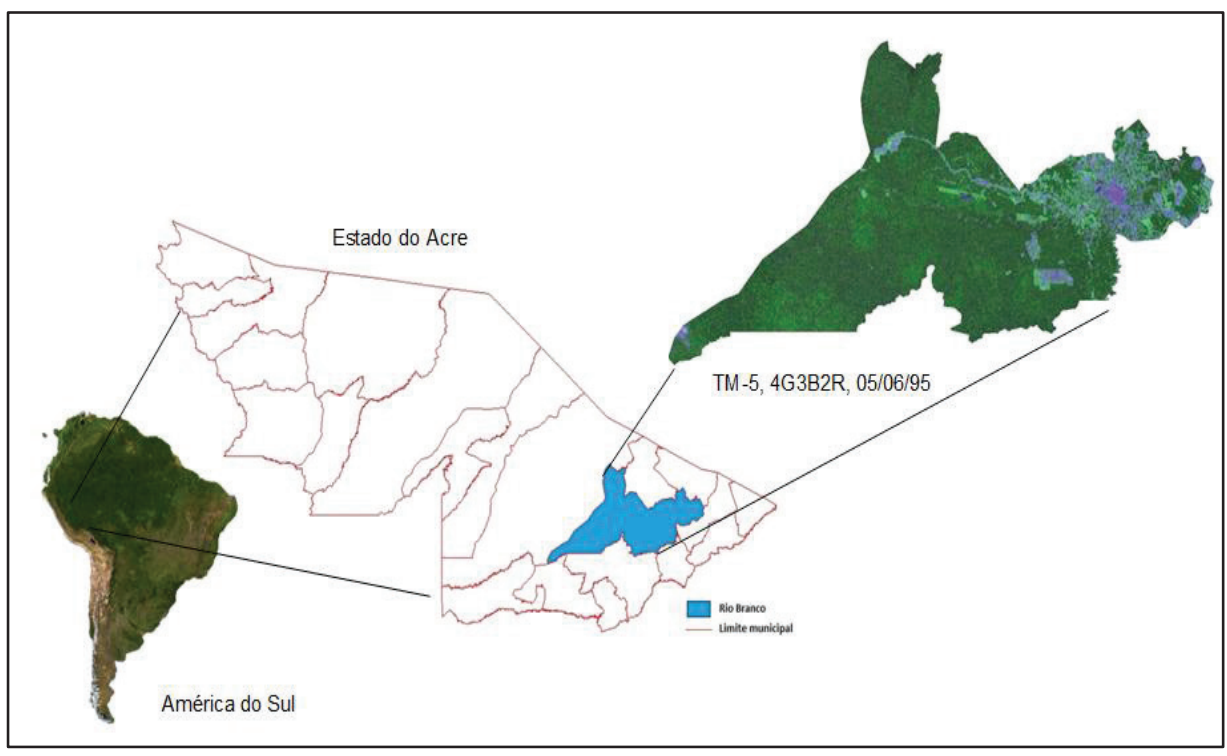

Figura 1 - Localização da área de estudo - Rio Branco (AC)

A precipitação média acumulada no mês oscila de 35,6 mm (agosto) a 293,2 $\mathrm{mm}$ (março), apresentando um total anual médio de 1963,4 $\mathrm{mm}$. Ocorrem duas estações distintas: uma chuvosa e uma menos chuvosa. A estação menos chuvosa inicia-se no mês de junho, prolongandose até o mês de outubro. A estação chuvosa inicia-se no mês de dezembro e estende-se até abril, caracterizada por chuvas constantes e abundantes. Os meses de maio e novembro podem ser caracterizados como meses de transicão da estacão chuvosa para a menos chuvosa e da menos chuvosa para a chuvosa, respectivamente.

Os principais sistemas meteorológicos que atuam na região e que contribuem para a formação de nuvens e chuvas, na estação chuvosa, são: mecanismos convectivos e de transporte de umidade a partir da evaporação no Oceano Atlântico e da evapotranspiração na Floresta Amazônica, nesta época abundam os cúmulos de grande desenvolvimento verticais. Para a estação seca a maior contribuição para 
eventuais chuvas advém de convecção local e frentes frias, procedentes da região Sul do Brasil, ocasionando o fenômeno denominado de friagens. Estas são resultantes do avanço de uma Frente Polar impulsionada por uma Massa de Ar Polar Atlântica que avança pela Planície do Chaco até a Amazônia Ocidental e provoca a queda de temperatura, até $10^{\circ} \mathrm{C}$, na região (FERRAZ, 2008; DUARTE, 2006). A umidade relativa do ar média mensal varia de $77,6 \%$ (agosto) a $87,6 \%$ (fevereiro), com média anual de $84,3 \%$

A velocidade média mensal do vento oscila de $0,99 \mathrm{~m} \mathrm{~s}-1$ em junho a $1,23 \mathrm{~m} \mathrm{~s}^{-1}$ (novembro), apresentando média anual de 1,1 m $\mathrm{s}^{-1}$. Durante a estação menos chuvosa (junho a novembro) predominam ventos do sul, sudeste e do leste, no entanto na época chuvosa predominam os ventos de norte e noroeste (DUARTE, 2006).

Para a insolação, que é o número de horas em que o sol brilha não oculto pelo céu encoberto de nuvens ou de fumaça em uma determinada região. Observa-se que os valores médios mensais para Rio Branco, AC, variam de um mínimo de 3,41 h em fevereiro a um máximo de 6,98 h em julho.

Segundo os critérios adotados por Thornthwaite, o clima da região de Rio Branco, classifica-se como Clima Úmido, com pequena deficiência de água, megatérmico e com vegetação durante todo o ano, representado pela seguinte fórmula climática: B1rA'a' (FERRAZ, 2008).

\section{Dados meteorológicos observacionais}

Os dados meteorológicos utilizados na presente pesquisa para o cálculo da Evapotranspiração potencial (ETp) foram extraídos do banco de dados do Instituto Nacional de Meteorologia - INMET (INMET, 2013) referente ao período de janeiro de 1980 a dezembro de 2013 (34 anos), medidos na estação meteorológica convencional $\mathrm{n}^{\mathrm{o}}$ 82915, instalada no município de Rio Branco (AC), nas dependências da Universidade Federal do Acre (UFAC), sito na BR-364, Km 04, nas coordenadas geográficas: $9^{\circ} 58^{\prime} 12^{\prime \prime} \mathrm{S}$ de latitude e $67^{\circ} 52^{\prime}$ $12^{\prime \prime} \mathrm{W}$ de longitude e $160 \mathrm{~m}$ de altitude. Utilizaram-se dados mensais de temperatura média, máxima e mínima do ar (Tmed, Tmax e Tmin, ${ }^{\circ} \mathrm{C}$ ), Umidade Relativa do ar (UR, \%),
Tmin, $\left.{ }^{\circ} \mathrm{C}\right)$, Umidade Relativa do ar (UR, \%), Pressão atmosférica (Pres., $\mathrm{kPa}$ ), velocidade do vento medida a 2 metros de altura $\left(\mathrm{u}_{2}, \mathrm{~m} \mathrm{~s}^{-1}\right)$, precipitação pluviométrica (prp, $\mathrm{mm}$ ) e insolação (n, horas).

\section{Consistência dos dados}

Entende-se por imputação a técnica de preencher os dados faltantes com valores plausíveis, desta forma utilizou-se no presente estudo o MTSDI (Multivariate time series data Imputation) que é um algoritmo para preenchimento de dados faltantes em séries temporais normais multivariadas baseados no algoritmo Expectation Maximisation (EM), proposto por Junger \& Leon (2012). Além da estrutura de correlação entre as estações levada em consideração na matriz de covariâncias dos dados, o método considera também a correlação temporal, por meio da modelagem independente das séries temporais em cada estação. Diversos estudos têm utilizado métodos multivariados de imputação para preencher dados faltantes em diversas áreas de pesquisas, tais como saúde e clima e concluíram que os procedimentos multivariados apresentaram melhor desempenho e acurácia que os univariados e que os métodos multivariados com ajuste do componente temporal apresentaram maior acurácia e precisão (COSTA et al., 2012; NUNES et al., 2010. JUNGER, 2008, NUNES, 2007).

A técnica foi utilizada para as oito variáveis meteorológicas do IMMET (Tabela 1) referente ao município de Rio Branco (AC) entre janeiro de 1980 a dezembro de 2013. Como variáveis preditoras, usaram-se séries temporais das variáveis meteorológicas da Rede de Meteorologia do Comando da Aeronáutica (REDEMET, 2014), disponível pelo Sistema de Geração e Disponibilização de Informações Climatológicas. As Tabelas 2 e 3, mostram as porcentagem total dos dados faltantes para o período e as coordenadas geográficas das estações e suas fontes, respectivamente.

Os dados faltantes que se deseja completar são caracterizados por "NA", e foi usado o número de imputações múltiplas padrão do pacote $m t s d i$ versão 2.15 do software estatístico $R$ de fácil utilização e páginas de ajuda ( $\mathrm{R}$ Core Team, 2013). A série da variável meteorológica original com falha é colocada lado a lado das estações mais próximas da localidade, em uma matriz exemplificada na Tabela 5. 
Tabela 1- Informações dos dados meteorológicos utilizados

\begin{tabular}{ccc}
\hline Variáveis meteorológicas & Unidade & Dados faltantes \\
\hline Tmed & ${ }^{\circ} \mathrm{C}$ & $18 \%$ \\
Tmin & ${ }^{\circ} \mathrm{C}$ & $11 \%$ \\
Tmax & ${ }^{\circ} \mathrm{C}$ & $20 \%$ \\
UR & $\%$ & $32 \%$ \\
Pressão & $\mathrm{hPa}$ & $26 \%$ \\
Precipitação & $\mathrm{mm}$ & $6 \%$ \\
Vel. Vento & $\mathrm{m} \mathrm{s}^{-1}$ & $10 \%$ \\
Insolação & horas & $7 \%$ \\
\hline
\end{tabular}

Tabela 2 - Informações das estações meteorológicas utilizadas

\begin{tabular}{lcc}
\hline \multicolumn{1}{c}{ Estação } & Fonte & Lat. - Lon. \\
\hline Rio Branco (AC) & INMET & $09^{\circ} 58^{\prime} \mathrm{S}-67^{\circ} 48^{\prime} \mathrm{W}$ \\
Cruzeiro do Sul (AC) & REDEMET & $07^{\circ} 36^{\prime} \mathrm{S}-72^{\circ} 46^{\prime} \mathrm{W}$ \\
Plácido de Castro (AC) & REDEMET & $09^{\circ} 58^{\prime} \mathrm{S}-67^{\circ} 48^{\prime} \mathrm{W}$ \\
Porto Velho (RO) & REDEMET & $08^{\circ} 42^{\prime} \mathrm{S}-63^{\circ} 54^{\prime} \mathrm{W}$ \\
\hline
\end{tabular}

Tabela 3 - Ilustração de dados a serem imputados, representados por NA, referentes à umidade relativa do ar para a estação de Rio Branco (a) e dados imputados (b). Os dados originais (coluna rbr) estão ao lado das séries das estações mais próximas (rbr-rede; crz-rede e rond-rede)

\begin{tabular}{|c|c|c|c|c|c|c|c|c|c|c|c|}
\hline \multicolumn{6}{|c|}{ (a) } & \multicolumn{6}{|c|}{ (b) } \\
\hline ano & mes & $\mathrm{rbr}$ & rbr-rede & crz-rede & rond-rede & & & & & & rond redo \\
\hline 1980 & 1 & 84,8 & 79,3 & 80,42 & 86,10 & $\begin{array}{l}\text { ano } \\
1980\end{array}$ & mes & $\begin{array}{l}\text { rbr } \\
\quad 84.80\end{array}$ & rbr-rede & $\begin{array}{c}\text { crz-rede } \\
80.42\end{array}$ & $\begin{array}{c}\text { rond-rede } \\
86.10\end{array}$ \\
\hline 1980 & 2 & 87,72 & 82 & 79,36 & 85,90 & 1980 & 2 & 87,72 & 82 & 79,36 & 85,90 \\
\hline 1980 & 3 & 89,91 & 81,6 & 78,93 & 86,40 & 1980 & 3 & 89,91 & 81,6 & 78,93 & 86,40 \\
\hline 1980 & 4 & 87,51 & 75,9 & 77,00 & 82,70 & 1980 & 4 & 87,51 & 75,9 & 77,00 & 82,70 \\
\hline 1980 & 5 & 86,78 & 76,8 & 76,28 & 80,10 & 1980 & 5 & 86,78 & 76,8 & 76,28 & 80,10 \\
\hline 1980 & 6 & 88,44 & 75,3 & 75,52 & 80,80 & 1980 & 6 & 88,44 & 75,3 & 75,52 & 80,80 \\
\hline 1980 & 7 & NA & 71,8 & 75,85 & 78,20 & 1980 & 7 & 82,74 & 71,8 & 75,85 & 78,20 \\
\hline 1980 & 8 & NA & 69,6 & 74,61 & 75,30 & 1980 & 8 & 81,50 & 69,6 & 74,61 & 75,30 \\
\hline 1980 & 9 & NA & 67,9 & 74,96 & 77,10 & 1980 & 9 & 81,40 & 67,9 & 74,96 & 77,10 \\
\hline 1980 & 10 & NA & 74,1 & 77,53 & 82,80 & 1980 & 10 & 85,56 & 74,1 & 77,53 & 82,80 \\
\hline 1980 & 11 & NA & 75,4 & 77,46 & 83,60 & 1980 & 11 & 86,95 & 75,4 & 77,46 & 83,60 \\
\hline 1980 & 12 & 85,32 & 76,3 & 79,43 & 85,90 & 1980 & 12 & 85,32 & 76,3 & 79,43 & 85,90 \\
\hline
\end{tabular}

\section{Evapotranspiração potencial}

A medição direta da Evapotranspiração (ET) é caracterizada pela determinação da ET diretamente na área de estudo e pode ser obtida, por exemplo, por meio das formas propostas pelos métodos do Lisímetro e Sondas de nêutrons (OMETTO, 1981).

Tais métodos são relativamente complicados de serem realizados, uma vez que se necessita de uma infraestrutura muito especifica e até certo ponto muito complexa para o fim que se propõem e ficam muito restritos a centros de pesquisas e tem a sua importância fundamental na calibração dos métodos indiretos para se estimar a ET (RUHOFF , 2011).

$\mathrm{Na}$ ausência de medidas diretas da ET, pesquisadores e irrigantes, muitas vezes, lançam mão de modelos físico-matemáticos (métodos indiretos) que se baseiam em elementos 
climáticos provenientes de estações meteorológicas, para proceder ao cálculo da Evapotranspiração potencial (ETp).

O método desenvolvido por PenmanMonteith (Equação 1) e, posteriormente, parametrizado pela FAO é conhecido atualmente como método Penman-Monteith-FAO (PMFAO). Este método é recomendado como padrão porque tem se mostrado consistente para estimar a ETp em diferentes locais e clima, além de considerar todos os parâmetros que governam as trocas de energia e o fluxo de calor latente. Outro aspecto é que a maioria dos parâmetros pode ser medido ou estimado a partir de dados meteorológicos (ALLEN et al., 1998). Outro aspecto é que a maioria dos parâmetros podem ser medidos ou estimados a partir de dados meteorológicos (ALLEN et al., 1998). Diversos trabalhos confirmam que o desempenho desse método é satisfatório, quando comparado com medidas em lisímetros: Jensen et al. (1990), Allen et al. (1994) em outros países, Camargo \& Sentelhas (1997), Pereira (1998), Santiago (2001) e Medeiros (2002) no Brasil. Todavia, em algumas situações, o emprego do método de PMFAO é restringido pela indisponibilidade de dados meteorológicos, o que acaba exigindo o uso de métodos mais simples.

A equação de PM-FAO é dada a seguir:

$E T p=\frac{0,408 \cdot \Delta\left(R_{n}-G\right)+\gamma\left(\frac{900 \cdot u_{2}}{T_{\text {med }}+273}\right)\left(e_{s}-e_{a}\right)}{\Delta+\gamma \cdot\left(1+0,34 u_{2}\right)}$

em que: ETp é a evapotranspiração potencial $\left(\mathrm{mm} \mathrm{dia}{ }^{-1}\right), \mathrm{R}_{\mathrm{n}}$ é a radiação líquida na superfície (MJ $\left.\mathrm{m}^{-2} \mathrm{dia}^{-1}\right), \mathrm{G}$ é o fluxo de calor do solo (MJ $\mathrm{m}^{-2}$ dia- $\left.^{-1}\right), \mathrm{T}_{\mathrm{med}}$ é a temperatura média diária do ar medida a 2 metros de altura $\left({ }^{\circ} \mathrm{C}\right)$, u2 é a velocidade do vento medida a 2 metros $\left(\mathrm{m} \mathrm{s}^{-1}\right)$, es é a pressão de vapor de saturação $(\mathrm{kPa})$, ), ea é a pressão atual de vapor $(\mathrm{kPa}), \Delta$ é a declividade da curva de pressão de vapor $\left(\mathrm{kPa}^{\circ} \mathrm{C}^{-1}\right)$ e $\gamma$ é a constante psicrométrica $\left(\mathrm{kPa}^{\circ} \mathrm{C}^{-1}\right)$.

As equações padronizadas e detalhes para o cálculo de todos os parâmetros da Equação (1) foram realizados conforme foram apresentados em Allen et al. (1998), Faria et al. (2003) e Fernandes (2010).
Variabilidade mensal, sazonal e interanual da Evapotranspiração potencial

No presente estudo foi aplicado o gráfico descritivo boxplot, descrito por Wilks (2011) para estabelecer a variabilidade mensal, sazonal e interanual da Evapotranspiração potencial para Rio Branco (AC). Esta metodologia inclui informações sobre a distribuição de um conjunto de dados com base em alguns de seus parâmetros descritivos: a mediana, o primeiro quartil e terceiro quartil, valores máximos e mínimos. Técnicas de suavização de dados, estatísticas descritivas (média, mediana, desvio padrão e coeficiente de variação), gráficos e tabelas foram produzidos para uma melhor organização e análise estatística dos mesmos. As fórmulas para o cálculo das estatísticas descritivas podem ser encontradas em Moretin e Bussab (2004). Nesses cálculos utilizaram-se o programa R-project (R Core Team, 2013).

\section{Teste de Homogeneidade}

Para a caracterização climática de uma região, há necessidade de informações meteorológicas confiáveis e homogêneas, pois uma falha na série temporal pode comprometer a análise e as interpretações dos dados.

A homogeneidade é uma questão importante para detectar a variabilidade dos dados. Em geral, quando os dados são homogêneos, isto significa que as medições dos dados são tomadas ao mesmo tempo com os mesmos instrumentos e ambientes, ou seja, quando as variações ocorrem apenas em função das condições do tempo e clima (LONGOBORDI \& VILLANI, 2010; MARTINEZ et al. 2011). No entanto, é uma tarefa difícil quando se trata de dados meteorológicos, pois tais alterações são sempre causadas por mudanças nas técnicas de medição e procedimentos de observação, características e estruturas de ambiente e localização das estações (COSTA \& SOARES, 2009).

Para testar a homogeneidade das séries dos parâmetros meteorológicos aqui preconizados, foi aplicado o teste de seqüência ou "run test" recomendado pela Organização Meteorológica Mundial (OMM), por meio de sua 
nota técnica no 81 (THOM, 1966). Segundo Back (2001), o teste de Run é um teste não paramétrico apresentado por Thom (1966), e é usado para avaliar se uma série ocorre aleatoriamente e consiste em fazer a contagem do número de oscilações acima e abaixo da mediana em uma série naturalmente ordenada e considerando o erro de estimação (e) obtido pela equação (2) e descrito por Casademont \& Villanova (2014) para amostras $<60$.

$$
e=\mp 2,08 \frac{s}{\sqrt{n}}
$$

onde, $e$ é o erro de estimação, $\mathrm{S}$ é o desvio padrão amostral e n o número de elementos da amostra.

O número de oscilações abaixo ou acima da mediana é chamado de Run, e a hipótese a ser testada é se o valor observado está dentro da faixa aceitável de uma distribuição considerada normal. Valores altos de Run indicam muitas oscilações e valores baixos indicam um desvio em relação à mediana durante o período de registro.

Back (2001) descreve o método considerando que se a seqüência contém $\mathrm{N}_{1}$ símbolos de um tipo e $\mathrm{N}_{2}$ símbolos de outro tipo (e $\mathrm{N}_{1}$ e $\mathrm{N}_{2}$ não são muito pequenos), a distribuição amostral do número de Runs totais pode ser aproximada pela distribuição normal com média dada por:

$$
E(u)=\frac{2 \mathrm{~N}_{1} \mathrm{~N}_{2}}{\mathrm{~N}_{1}+\mathrm{N}_{2}}+1
$$

e a variância da distribuição pode ser estimada por

$$
\operatorname{Var}(\mathrm{u})=\frac{2 \mathrm{~N}_{1} \mathrm{~N}_{2}\left(2 \mathrm{~N}_{1} \mathrm{~N}_{2}-\mathrm{N}_{1}-\mathrm{N}_{2}\right)}{\left(\mathrm{N}_{1}+\mathrm{N}_{2}\right)^{2}\left(\mathrm{~N}_{1}+\mathrm{N}_{2}-1\right)}
$$

em que " $u$ " representa o número de Runs. Por isso, a hipótese nula de que a distribuição dos símbolos ocorre normalmente e que a amostra é aleatória, pode ser testada com base na estatística:

$$
\mathrm{R}=\frac{\mathrm{u}-\mathrm{E}(\mathrm{u})}{\sqrt{\operatorname{Var}(\mathrm{u})}}
$$

Este valor calculado pode ser comparado aos valores teóricos da distribuição normal $R$ para distribuição normal, considerando o nível de significância de 5\%, R deve estar entre -1,96 e 1,96. Caso $\mathrm{R}$ calculado seja maior que estes limites tabelados, rejeita-se a hipótese de nulidade.

\section{Resultados e Discussão}

A Tabela 4 mostra o número de Runs (u), o valor da estatística $R, O$ valor critico de $R\left(R_{c}\right)$ e os resultados obtidos segundo a metodologia descrita no item Teste de Homogeneidade. Observase que dentre os nove parâmetros meteorológicos analisados pelo referido teste todas as séries de dados foram consideradas como homogêneas estando os valores da estatística $\mathrm{R}$ (em módulo) variando entre 0,18 a 1,38, ou seja, baixo do valor crítico de $|1,96|$. Assim, portanto, a série histórica de Evapotranspiração potencial, que é o objeto principal deste estudo, assim como as variáveis meteorológicas utilizadas para a sua estimativa, pode ser usada para estudos mais detalhados que envolva análises de tendência de séries temporais na localidade de Rio Branco, Acre.

Tabela 4 - Resultados do teste de seqüência (Run test) para as séries anuais dos parâmetros meteorológicos para Rio Branco (AC) - 1980 a 2013

\begin{tabular}{lcccc}
\hline \multicolumn{1}{c}{ Variável } & $\begin{array}{c}\mathrm{N}^{\circ} \text { de Runs } \\
(\mathrm{u})\end{array}$ & $\begin{array}{c}\text { Estatística } \\
|\mathrm{R}|\end{array}$ & $\begin{array}{c}\text { Valor crítico } \\
\left|\mathrm{R}_{\mathrm{c}}\right|\end{array}$ & Resultado \\
\hline Temperatura média & 12 & 0,77 & 1,96 & $\mathrm{H}$ \\
Temperatura máxima & 12 & 0,77 & 1,96 & $\mathrm{H}$ \\
Temperatura mínima & 08 & 1,38 & 1,96 & $\mathrm{H}$ \\
Umidade relativa & 14 & 0,19 & 1,96 & $\mathrm{H}$ \\
Insolação & 15 & 0,54 & 1,96 & $\mathrm{H}$ \\
Pressão atmosférica & 11 & 0,05 & 1,96 & $\mathrm{H}$ \\
Velocidade do vento & 10 & 1,15 & 1,96 & $\mathrm{H}$ \\
Precipitação pluviométrica & 13 & 0,20 & 1,96 & $\mathrm{H}$ \\
Evapotranspiração potencial & 15 & 0,18 & 1,96 & $\mathrm{H}$ \\
\hline
\end{tabular}


Análise mensal, sazonal e interanual da Evapotranspiração potencial diária.

Regionalmente, verifica-se que em Rio Branco (AC) por meio da Figura 2 e Tabela 5 que os valores médios de ETp revelam uma variação sazonal bastante forte, com os seguintes valores diários médios mensais aproximados: um máximo de $4,13 \mathrm{~mm} \mathrm{~d}^{-1}$ em outubro a um mínimo de 2,93 $\mathrm{mm} \mathrm{d}^{-1}$ em junho, para uma média anual de $3,54 \mathrm{~mm} \mathrm{~d}^{-1}$ com um acumulado médio anual de 1290,99 mm. Contudo valores mais elevados/baixos podem ocorrer ao longo do ano, conforme pode ser observado pelos valores máximos e mínimos da Figura 2 e mostrados pela Tabela 5 registrados para o período de estudo. Esta distribuição sazonal da ETp na região está associada a nebulosidade advinda da migração SE/NW da convecção amazônica que é controlada pela disponibilidade de energia em que medidas realizada na Amazônia Central (Manaus-AM) indicaram que os maiores totais de radiação que chegam na superfície ocorrem nos meses de setembro/outubro, sendo que os mínimos são nos meses de dezembro à fevereiro (HOREL, et al., 1989)

Os valores da média ao longo do ano são próximos da mediana (Tabela 5), indicando que a média é um bom estimador para representar a ETp para a localidade. Com relação à variabilidade, observa-se que os valores dos Desvios Padrões (D.P.) oscilam em cerca de 7,5\% dos seus respectivos valores médios, sendo junho e agosto os meses com maiores variabilidade 9,1 e 9,8\%, respectivamente, o trimestre outubro, novembro, dezembro e os meses janeiro, fevereiro os menores ( $6 \%)$. Assim, a ETp em qualquer mês do ano pode ser, em média $\sim 7,5 \%$ maior ou menor do que o seu valor médio esperado.

Resultados semelhantes foram observados por Ferraz (2008) e Souza (2009) para Rio Branco (AC) comparando-se métodos mais simples para estimativa da Evapotranspiração de referencia com os dados determinados pelo método padrão recomendado pela FAO (PMFAO) para um período de 25 anos (1981 a 2006), em que observaram que a média anual oscilou em torno de $3,39 \mathrm{~mm} \mathrm{~d}^{-1}$ para um acumulado anual de 1237,35 mm, com máximo mensal para o mês de outubro $\left(3,96 \mathrm{~mm} \mathrm{~d}^{-1}\right)$ e mínimo em junho $\left(2,79 \mathrm{~mm} \mathrm{~d}^{-1}\right)$.

Hasler e Avissar (2006) com objetivo de caracterizar a variabilidade espacial e temporal da ET utilizando fluxo de vórtices turbulentos de oito torres micrometeorológicas espalhas pela Amazônia conclui que as estações mais próximas do equador $\left(2^{\circ}\right.$ a $3^{\circ}$ S) mostraram forte sazonalidade, aumentando na estação seca (junset) e diminuindo na estação chuvosa (dez-mar) atribuindo a sazonalidade ao ciclo anual da radiação solar e as estações mais ao sul da Amazônia $\left(9^{\circ}\right.$ a $\left.11^{\circ} \mathrm{S}\right)$ sem sazonalidade clara, porém paras essas estações mais ao sul o saldo de radiação é o parâmetro que mais se correlacionou com a ET que é comumente associada com o estresse hídrico.

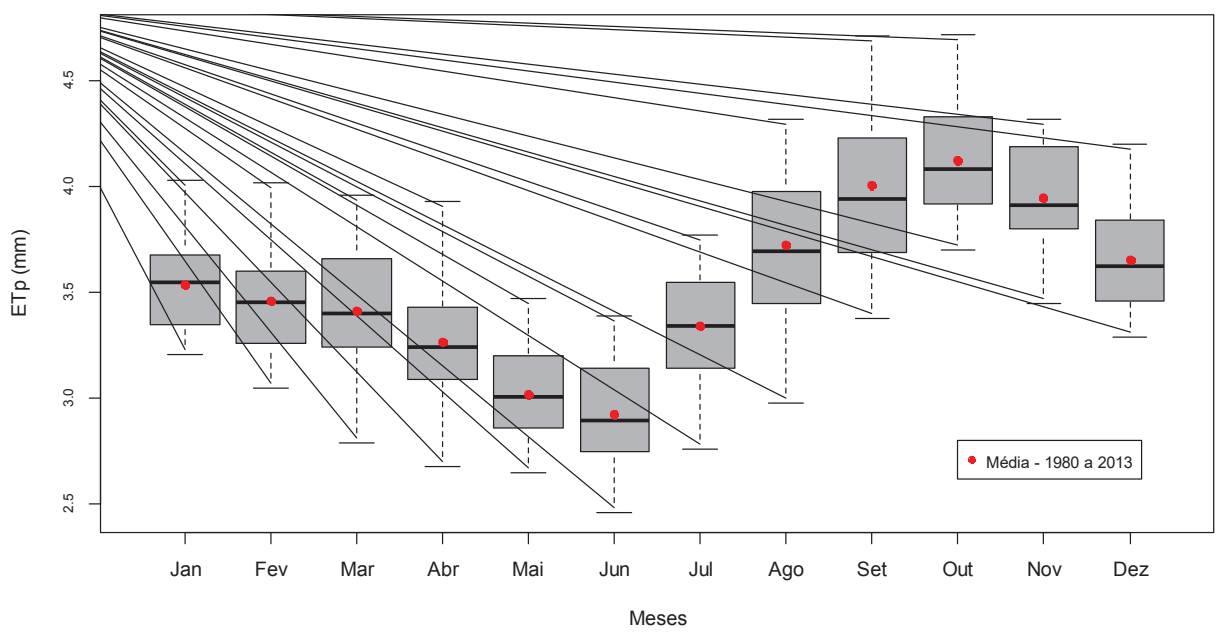

Figura 2 - Boxplot dos valores médios mensais de Evapotranspiração potencial, ETp $\left(\mathrm{mm} \mathrm{d}^{-1}\right)$, para Rio Branco (AC) - 1980 a 2013 
Tabela 5 - Estatística descritiva da série histórica dos valores médios mensais de Evapotranspiração potencial, $\operatorname{ETp}\left(\mathrm{mm} \mathrm{d}^{-1}\right)$ para Rio Branco (AC) - 1980 a 2013

\begin{tabular}{lccccccccc}
\hline \multicolumn{1}{c}{ Meses } & $\begin{array}{c}\text { Valor } \\
\text { Mín. }\end{array}$ & $\begin{array}{c}\text { Q1. } \\
(\mathrm{mm})\end{array}$ & $\begin{array}{c}\text { Mediana } \\
(\mathrm{mm} / \mathrm{dia})\end{array}$ & $\begin{array}{c}\text { Média } \\
(\mathbf{m m} / \mathbf{d i a})\end{array}$ & $\begin{array}{c}\text { Q3. } \\
(\mathrm{mm})\end{array}$ & $\begin{array}{c}\text { Valor } \\
\text { Máx. }\end{array}$ & $\begin{array}{c}\text { D.P. } \\
(\mathrm{mm})\end{array}$ & $\begin{array}{c}\text { C.V. } \\
(\%)\end{array}$ & $\begin{array}{c}\text { ETp } \\
(\mathbf{m m} / \mathbf{m e ̂ s})\end{array}$ \\
\hline Janeiro & 3,21 & 3,36 & 3,56 & $\mathbf{3 , 5 4}$ & 3,68 & 4,03 & 0,21 & 6,0 & $\mathbf{1 0 9 , 6 7}$ \\
Fevereiro & 3,05 & 3,26 & 3,46 & $\mathbf{3 , 4 6}$ & 3,60 & 4,02 & 0,23 & 6,6 & $\mathbf{9 6 , 9 3}$ \\
Março & 2,79 & 3,42 & 3,40 & $\mathbf{3 , 4 2}$ & 3,66 & 3,96 & 0,26 & 7,6 & $\mathbf{1 0 5 , 9}$ \\
Abril & 2,68 & 3,11 & 3,24 & $\mathbf{3 , 2 7}$ & 3,43 & 3,93 & 0,26 & 8,1 & $\mathbf{9 7 , 9 8}$ \\
Maio & 2,65 & 2,87 & 3,01 & $\mathbf{3 , 0 2}$ & 3,18 & 3,47 & 0,22 & 7,2 & $\mathbf{9 3 , 6 6}$ \\
Junho & 2,46 & 2,75 & 2,90 & $\mathbf{2 , 9 3}$ & 3,14 & 3,39 & 0,27 & 9,1 & $\mathbf{8 7 , 8 6}$ \\
Julho & 2,76 & 3,16 & 3,34 & $\mathbf{3 , 3 4}$ & 3,55 & 3,77 & 0,27 & 8,0 & $\mathbf{1 0 3 , 5 8}$ \\
Agosto & 2,98 & 3,46 & 3,70 & $\mathbf{3 , 7 3}$ & 3,98 & 4,32 & 0,37 & 9,8 & $\mathbf{1 1 5 , 4 8}$ \\
Setembro & 3,38 & 3,72 & 3,94 & $\mathbf{4 , 0 1}$ & 4,23 & 4,71 & 0,35 & 8,8 & $\mathbf{1 2 0 , 1 9}$ \\
Outubro & 3,70 & 3,93 & 4,09 & $\mathbf{4 , 1 3}$ & 4,32 & 4,72 & 0,28 & 6,7 & $\mathbf{1 2 7 , 9 1}$ \\
Novembro & 3,45 & 3,80 & 3,91 & $\mathbf{3 , 9 5}$ & 4,18 & 4,32 & 0,24 & 6,1 & $\mathbf{1 1 8 , 5}$ \\
Dezembro & 3,29 & 3,47 & 3,63 & $\mathbf{3 , 6 6}$ & 3,84 & 4,20 & 0,22 & 6,2 & $\mathbf{1 1 3 , 3 3}$ \\
\hline
\end{tabular}

Na Figura 3 e Tabela 6 que apresentam os valores da estatística descritiva (média, mediana, valores máximos, mínimos e as separatrizes) em escala sazonal (estações do ano), observa-se que a ETp pode ser caracterizada como simétrica em torno dos seus valores médios: $3,55 \mathrm{~mm} \mathrm{~d}^{-1}$ no verão austral (DJF), de $3,23 \mathrm{~mm} \mathrm{~d}^{-1}$ no outono austral (MAM), 3,33 mm $\mathrm{d}^{-1}$ no inverno austral (JJA) e de $4,03 \mathrm{~mm} \mathrm{~d}^{-1}$ na primavera austral (SON), o que nos remete a um nítido efeito de hierarquia sazonal. Ao avaliar e comparar a variabilidade de conjunto de dados através da Figura 3 e Tabela 6, observa-se que o outono possui, em geral, valores de ETp menores e mais homogêneos quanto comparado como os valores médios da primavera que apresentou os maiores valores e também maior variabilidade. Observa-se que $25 \%$ dos valores da primavera estão abaixo de $3,38 \mathrm{~mm} \mathrm{~d}^{-1}$ e $25 \%$ acima de 4,22 $\mathrm{mm} \mathrm{d}^{-1}$. Para o outono $25 \%$ dos valores estão abaixo $2,65 \mathrm{~mm} \mathrm{~d}^{-1}$ e $25 \%$ acima de $3,42 \mathrm{~mm} \mathrm{~d}^{-1}$ (Tabela 6). A estação do ano que apresentou maior variabilidade foi o inverno com um Desvio Padrão (D.P.) de $0,43 \quad \mathrm{~mm} \mathrm{~d}^{-1}$ e Coeficiente de Variação (C.V.) de $12,8 \%$ e a menor variabilidade para estação de verão com um D.P. de $0,24 \mathrm{~mm} \mathrm{~d}^{-1}$ e C.V. de $9 \%$. A máxima ETp assinalada para os meses de primavera austral pode está associada ao maior aquecimento radioativo devido a presença de poucas nuvens, característicos para essa época do ano na região. Os meses de outono austral, ao contrário, apresentam os menores valores de ETp em virtude, é claro, da menor forçante evapotranspirativa - o aquecimento por radiação solar e pela incursão de friagens que fazem a temperatura média diminuir consideravelmente na localidade. 


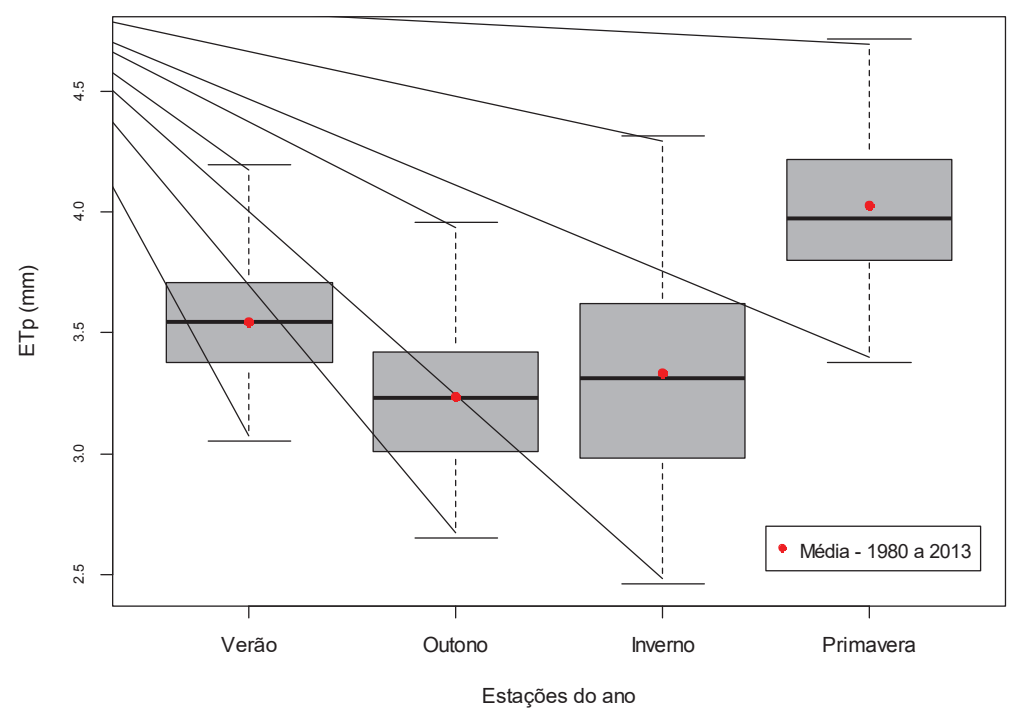

Figura 3 - Boxplot sazonal da ETp diária para o município de Rio Branco, AC - 1980 a 2013

Tabela 6 - Estatística descritiva da série histórica da média diária sazonal da ETp para Rio Branco, AC - 1980 a 2013

\begin{tabular}{lcccc}
\hline \multicolumn{1}{c}{ Estatísticas } & Verão & Outono & Inverno & Primavera \\
\hline Valor Mín. & 3,05 & 2,65 & 2,46 & 3,38 \\
$\mathrm{Q}_{1}$ & 3,38 & 3,01 & 3,00 & 3,80 \\
Mediana & 3,55 & 3,23 & 3,35 & 3,98 \\
Média & 3,55 & 3,23 & 3,33 & 4,03 \\
$\mathrm{Q}_{3}$ & 3,70 & 3,42 & 3,62 & 4,22 \\
Valor Máx. & 4,20 & 3,96 & 4,32 & 4,72 \\
D.P $(\mathrm{mm})$ & 0,24 & 0,29 & 0,43 & 0,31 \\
C.V.(\%) & 6,7 & 8,8 & 12,8 & 7,8 \\
\hline
\end{tabular}

Para Rio Branco (AC) observa-se na Figura 4 e mostrados na Tabela 7 que os valores médios do total anual da ETp ao longo do período analisado (1980 a 2013) foi de 107,58 mm mês ${ }^{-1}$ (linha tracejada azul) com um desvio padrão de $4,45 \mathrm{~mm}$ mês $^{-1}$ e para o acumulado anual de 53,41 mm ano ${ }^{-1}$ com média de 1290,99 $\mathrm{mm}$ ano $^{-1}$ (com C.V. de 4,1\%). De acordo, ainda, com a Figura 4, é possível verificar que, os anos com maiores demandas evapotranspirativas foram de 2005 a 2013, com um valor médio de $113,20 \mathrm{~mm} \mathrm{mês}^{-1}$, sendo o ano de 2005, o de maior valor com 115,13 mm mês-1 $(1381,60 \mathrm{~mm}$ ano $^{-1}$ ) e o de menor valor ocorreu em $1993 \mathrm{com}$ $101,04 \mathrm{~mm}$ mês-1 $\left(1212,50 \mathrm{~mm}\right.$ ano $\left.^{-1}\right)$. Com relação à dispersão interanual observadas por meio das caixas interquartílicas do gráfico boxplot para cada ano, observa-se que o ano de 1993 foi o ano que apresentou menor dispersão e o ano de 2009, o de maior entre os dados observados. Valores discrepantes (outlines) são observados na série de dados, um para o ano de 1996 que ocorreu no mês de julho com um valor de $73,8 \mathrm{~mm}^{\text {mês }}{ }^{-1}$ muito abaixo da média que é $103,58 \mathrm{~mm}$ mês $^{-1}$. Os outros dois outlines ocorrem no ano de 2010 nos meses de outubro e novembro com os respectivos valores 134,5 e $135,3 \mathrm{~mm}$ mês $^{-1}$ que superaram as médias de 127,91 e a $118,50 \mathrm{~mm} \mathrm{mês}^{-1}$, respectivamente. Xu et al. (2014) estudando efeitos de fatores climáticos e as repostas dos ecossistemas sobre a variabilidade inter-anual da ET em uma plantação de coníferas na China, concluíram que os valores da ET que foram calculados de acordo com os pressupostos de que tanto o clima quando os ecossistemas são variáveis entre os anos, sugerem que a variabilidade inter-anual da ET foi dominado por respostas dos ecossistemas 
e que houve uma interação negativa entre os efeitos do clima e dos ecossistemas. Estes resultados sugerem que, para a localidade de estudo as respostas dos ecossistemas devem ser levados em consideração para um melhor entendimento da variabilidade da ET.

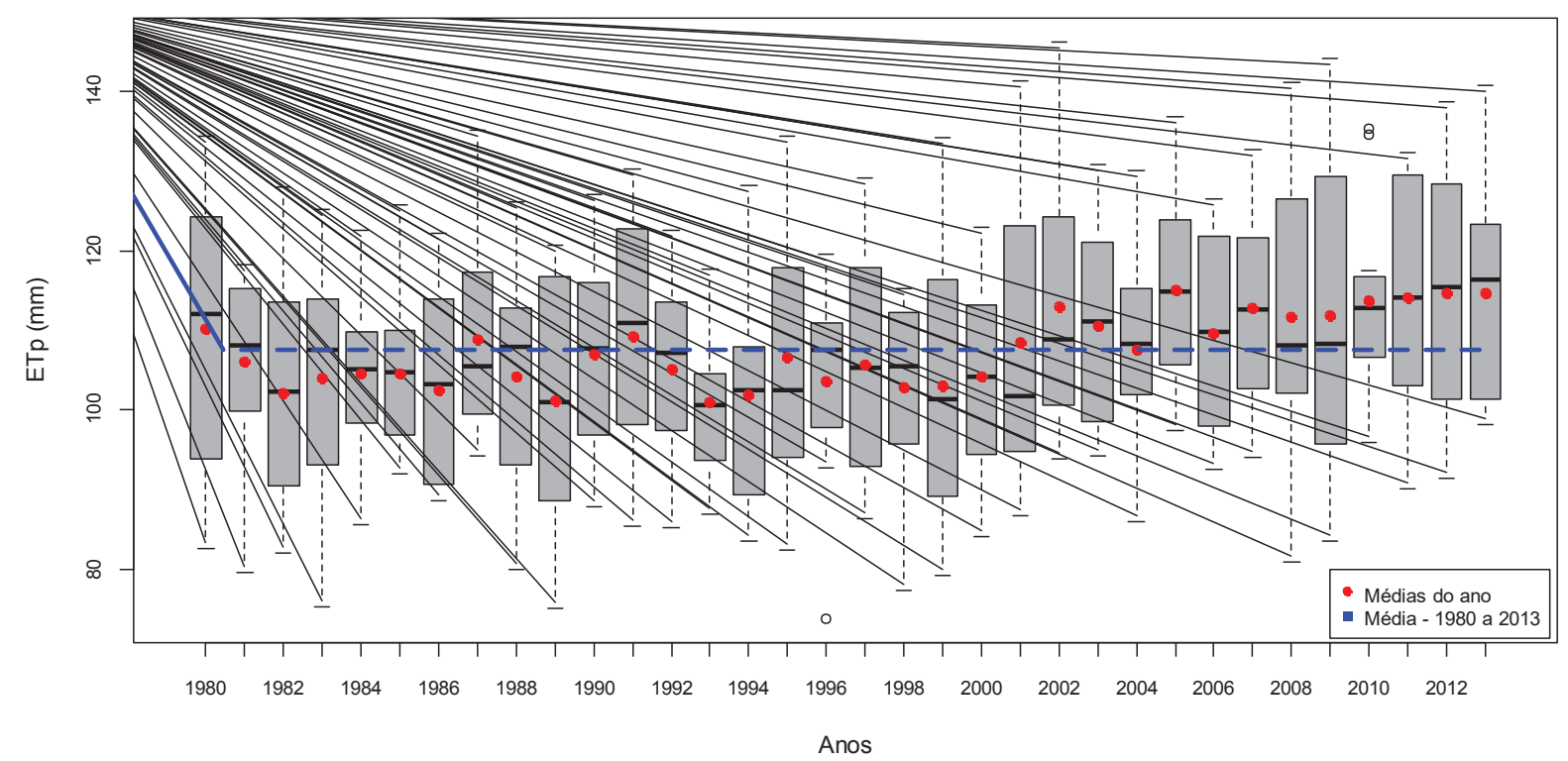

Figura 4 - Boxplot do acumulado médio mensal da ETp (mm mês $\left.{ }^{-1}\right)$ para Rio Branco, AC - 1980 a 2013

Tabela 7 - Estatística descritiva para a série histórica do acumulado médio anual da ETp para Rio Branco, AC 1980 a 2013

\begin{tabular}{lc}
\hline \multicolumn{1}{c}{ Estatísticas } & ETp (mm/ano) \\
\hline $\mathrm{Q}_{1}$ & 1247,93 \\
Valor Min. & 1212,5 \\
Mediana & 1281,33 \\
Média & $\mathbf{1 2 9 0 , 9 9}$ \\
$\mathrm{Q}_{3}$ & 1336,53 \\
Valor Max. & 1381,6 \\
D.P. (mm) & 53,41 \\
C.V. (\%) & 4,10 \\
\hline
\end{tabular}

A fim de corroborar com as informações obtidas pelo gráfico boxplot mensal, foi feita a decomposição da série temporal da ETp (mm dia $^{-1}$ ). Morrettin e Toloi (2006) consideram uma serie temporal descrevendo-as como uma soma de três componentes não-observáveis: tendência, sazonalidade e componente aleatória (ruídos) de média zero e variância constante. Observa-se por meio da Fig. 5 que a série temporal, visualmente, apresenta tendência crescente a partir do ano 2000, sazonalidade bem definida e ruídos aleatórios apresentando um comportamento estável dentro de um limite considerável. 


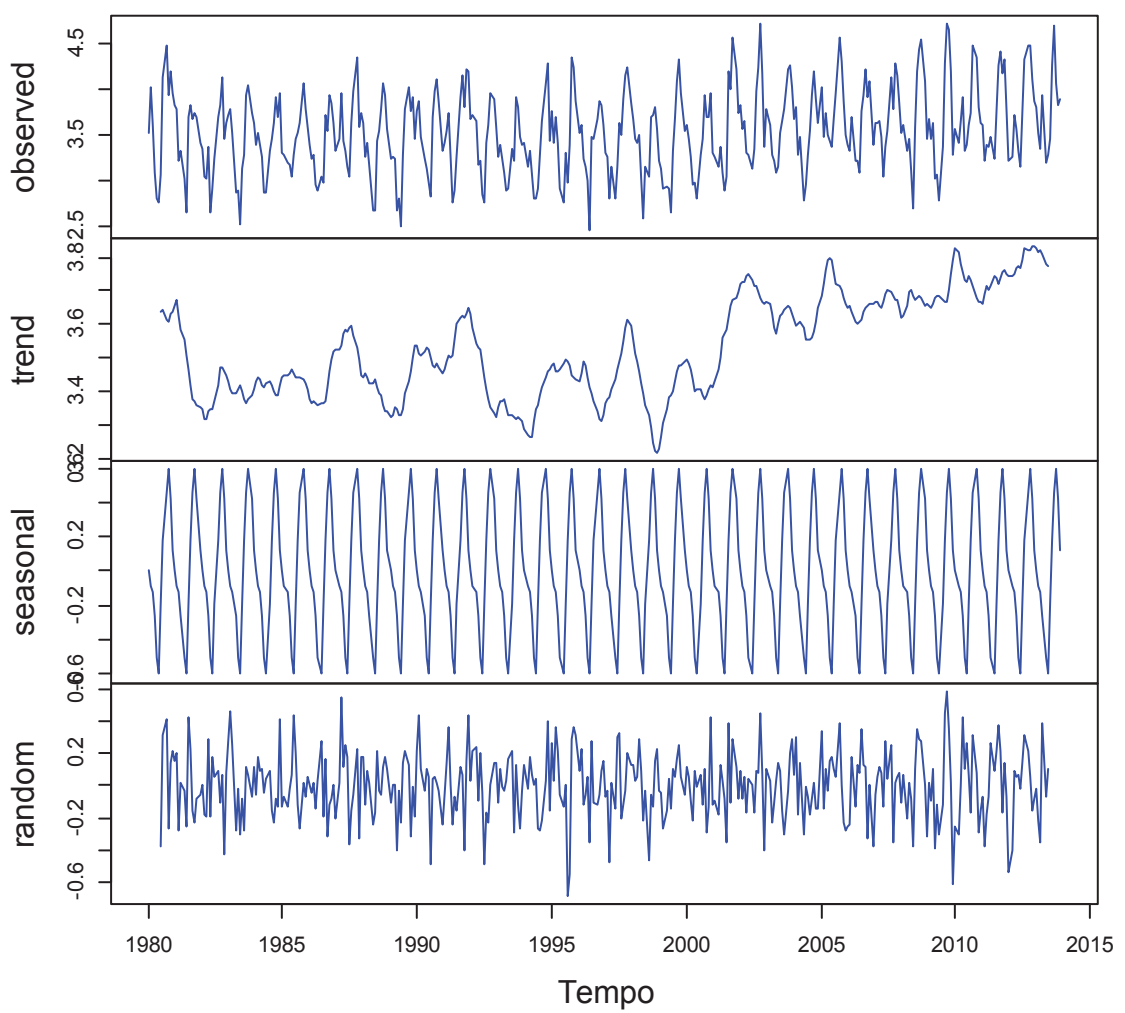

Figura 5 - Decomposição da série temporal da ETp ( $\left.\mathrm{mm} \mathrm{d}^{1}\right)$ em suas componentes tendência (trend), sazonal (seasonal) e ruídos (random) para o município de Rio Branco (AC) - 1980 a 2013

\section{Conclusões}

Os resultados deste trabalho permitem concluir que a média anual da ETp é de 3,54 mm $\mathrm{d}^{-1}$, com uma sazonalidade bastante marcante apresentando um mínimo em junho de 2,93 mm $\mathrm{d}^{-1}$ e um máximo no mês de outubro de 4,13 mm $\mathrm{d}^{-1}$ com um acumulado médio anual de 1290,99 mm e Desvio Padrão (D.P) de 53,41 mm.

Os anos com maiores demandas evapotranspirativas foram de 2005 a 2013, com um valor médio de $113,20 \mathrm{~mm} \mathrm{mês}^{-1}$, e o de menor o ano de 1993 (101,04 mm mes $\left.{ }^{-1}\right)$. Com relação à dispersão interanual observadas para cada ano, observa-se que o ano de 1993 foi o ano que apresentou menor dispersão e o ano de 2009, o de maior entre os dados observados.

A variabilidade sazonal da ETp na região de estudo considerando o D.P é menor nas estações verão e outono (período chuvoso) do que nas estações inverno primavera (período menos chuvoso) e que o maior valor do coeficiente de variação (C.V) é o do inverno que não está associado ao maiores valores de ETp que ocorrem na primavera.

Os valores baixos de ETp nos meses de outono e inverno, em relação aos registrados no verão e primavera, podem ser atribuídos a queda da temperatura média associada à entrada de frentes frias na região, o qual é denominado localmente de friagem.

\section{Agradecimentos}

A CAPES - Coordenação de Aperfeiçoamento de Pessoal de Nível Superior por meio da Fundação de Amparo a Pesquisa do estado do Acre (FAPAC), pela concessão da bolsa de estudo; ao programa de Pós-Graduação em Ciências Climáticas/UFRN pelo incentivo a pesquisa.

Ao INMET - Instituto Nacional de Meteorologia, pelos dados meteorológicos disponibilizados por meio do Banco de Dados Meteorológicos para Ensino e Pesquisa BDMEP. 


\section{Referências}

ALLEN, R. G.; SMITH, M.; PERRIER, A. An update for the definition of reference evapotranspiration. ICID BULLETIN, v. 43, n. 2, p.1-34, 1994.

ALLEN, R. G.; PEREIRA, L. S.; RAES, D.; SMITH, M. Crop evapotranspiration: guidelines for computing crop water requirements. Rome: FAO Irrigation and Dranaige Paper, 5), 1998.

ALENCAR, L. P.; SEDIYAMA, H. C.; WANDERLEY, H. S.; ALMEIDA, T. S.; DELGADO, R. C.; VIEIRA, G. H. S. Evolução temporal da evapotranspiração de referencia e sua sensibilidade ais principais elementos climáticos na cidade de Uberaba - MG. Revista Brasileira de Ciências Agrárias. v.7, n.1, p.x-xx, jan-mar., 2012.

BACK, A.J. Aplicação de análise estatística para identificação de tendências climáticas. Pesquisa Agropecuária Brasileira, v.36, p.717-726, 2001.

BROW, et al. Brazil: Drought and Fire Response in the Amazon. World Resources Report: Decision Making in a Changing Climate (http://www.worldresourcesreport.org/). 2011.

CASADEMONT, M.; VILLANOVA, R. Anàlisi pluviomètrica al mas Rusques en els anys 1982 2003, Acessado em $<$ http://www.econ.upf.es/ villanov/public-files/regimpluv.pdf $>$, julho de 2014.

CAMARGO, A. P.; SENTELHAS, P. C. Avaliação do desempenho de diferentes métodos de estimativa da evapotranspiração potencial no Estado de São Paulo, Brasil. Revista Brasileira de Agrometeorologia, Santa Maria, RS, v. 5, n. 1, p.89- 97, 1997.

CARVALHO, L. G.; RIOS, G. F. A.; MIRANDA, W. L.; NETO, P. C. Evapotranspiração de referência: Uma abordagem atual de diferentes métodos de estimativas. Pesq. Agropec. Trop., Goiânia, v. 41, n.3, p. 456-465, jul./set. 2011.

COSTA, A. C.; SOARES, A. Trends in extreme precipitation indices derived from a daily rainfall database for the South of Portugal. International Journal of Climatology, v. 29, p.1956-1975, 2009.

COSTA, R. L.; SILVA, F. D. S.; SARMANHO, G. F.; LUCIO, P. S. Imputação Multivariada de Dados Diários de Precipitação e Análise de Índices de Extremos Climáticos. Revista Brasileira de Climatologia Física, 03 (2012) 661675, 2012.

DUARTE, A. F. Aspectos da climatologia do acre, Brasil, com base no intervalo 1971 - 2000. Revista Brasileira de Meteorologia, v.21, n.3b, 308-317, 2006.

FERNANDES, R.; KOROLEVYCH, V.; WANG, $S$. Trends in Land Evapotranspiration over Canada for the Period 1960-2000 based on In Situ Climate Observations and a Land Surface Model. Journal of Hydrometeorology, Vol. 8, 2007.

FERRAZ, P. A. Estimativa da Evapotranspiração de referência (ETo) para a região de Rio Branco - ACRE. Dissertação de Mestrado (Programa de Pós-Graduação em Agronomia-Produção Vegetal) - Universidade Federal do Acre, 2008.

FARIA, R. T.; CAMARORI, P. H.; CHIBANA, E. Y.; BRITO, L. R. S. CLIMA - Programa computacional para organização e análise de dados meteorológicos. Eng. Agric., Jabocatibal, v. 23, n.2, p.372-387, maio/ago. 2003.

FERNANDES, D. S.; HEINEMANN, A. B.; AMORIM, A. O. Evapotranspiração - Uma Revisão sobre os Métodos Empíricos. Documentos 263. Embrapa Arroz e Feijão, Santo Antônio de Goiás, GO. 2010.

IBGE - INSTITUTO BRASILEIRO DE GEOGRAFIA E ESTATISTICA. IBGE Cidades. 
Rio Branco. 2010. Disponível em: http://ibge.gov.br/cidadesat. Acesso em: $14 \mathrm{fev}$. 2014.

INMET! Instituto Nacional de Meteorologia. Disponível em: http://www.inmet.gov.br/portal/.

JUNGER, W. \& LEON, A. P. mtsdi: Multivariate time series data imputation. $\mathrm{R}$ package version 0.3.3. http://CRAN.R-project.org/package=mtsdi, 2012.

JUNGER, W. L. Análise: Imputação de dados e interfaces computacionais em estudos de series temporais epidemiológicos. Rio de Janeiro, RJ, 2008. Tese (Doutorado em Medicina Social) Universidade do Estado do Rio de Janeiro. 2008

LONGOBARDI, A.; VILLANI, P. Trend analysis of annual and seasonal rainfall time series in the Mediterranean area. International Journal of Climatology, v.30, p.1538-1546, 2010.

HASLER, N. \& AVISSAR, R. What Controls Evapotranspiration in the Amazon Basin? Journal Of Hydrometeorology, vol. 8, 2006.

HOREL, J.D.; HAHMANN, A.N.; GEISLER, J.E. An investigation of the annual cycle of convective activity over the tropical Americas. Journal of Climate, 2(11), 1388 - 1403, 1989;

MARQUES, J.; SALATI, E.; SANTOS, J. M. Cálculo da evapotranspiração real na Bacia Amazônica através do método aerológico. Acta Amazônica. 10(2): 357-361, 1980.

MARTÍNEZ, M. D.; Serra, C.; Burgueño, A.; Lana, X. Short communication response to the comments on time trends of daily Maximum and minimum temperatures in Catalonia (NE Spain) for the period 1975-2004. International Journal of Climatology, v.3, p.153-157, 2011.
MEDEIROS, A. T. Estimativa da evapotranspiração de referência a partir da equação de Penman-Monteith, de medidas lisimétricas e de equações empíricas, em Paraipaba, CE. 2002. 103 f. Tese (Doutorado em Agronomia) - Escola Superior de Agricultura "Luiz de Queiroz", Universidade de São Paulo. Piracicaba, SP, 2002.

MORETTIN, P .A; TOLOI, C. M. Analise de series temporais - $2^{\underline{a}}$ Ed. Ver. Ampl. - São Paulo: Blucher, 2006.

MORETTIN, P. A.; BUSSAB, W. O. Estatística Básica, São Paulo: Editora Atlas, 2004.

NUNES, L. N.; KLUCK, M. M.; FACHEL, J. M. G. Comparação de métodos de imputação única e múltipla usando como exemplo um modelo de risco para mortalidade cirúrgica. Revista Brasileira Epidemol, noํ256, 13(4): 596-606, 2010.

NUNES, L. N. Métodos de imputação de dados aplicados na área da saúde. Tese de doutorado em medicina: Epidemiologia. Faculdade de medicina. Universidade federal do Rio Grande do Sul, Porto Alegre. 2007.

PAIVA, E. M. C. D.; CLAKE R. T. Análise de Tendência de Precipitação da Amazônia. Revista Brasileira de Meteorologia, 10 (1/2): 37-41, 1995.

PEREIRA, A. R. Introdução à micrometeorologia. Piracicaba, SP: ESALQ. Departamento de Física e Meteorologia, 1998. 70 p.

REDEMET. Homepage oficial do Comando Aéreo da Aeronáutica. Disponível em: http://clima.icea.gov.br/clima/. Acesso em: março de 2014

$\mathrm{R}$ Core Team. R: A language and environment for statistical computing. $\mathrm{R}$ Foundation for Statistical Computing, Vienna, Austria. ISBN 3- 
900051-07-0, URL http://www.R-project.org/ 2013.

RUHOFF, A. L. Sensoriamento Remoto aplicado à estimativa da evapotranspiração em Biomas Tropicais. Tese de doutorado (Programa de Pós-Graduação em Recursos Hídricos e Saneamento Ambiental), UFRS, Porto Alegre, RS, Brasil, 2011.

SANTIAGO, A. V. Evapotranspiração de referência medida por lisímetro de pesagem e estimada por Penman-Monteith (FAO-56), nas escalas mensal e decendial. 2001. 37 p. Dissertação (Mestrado em Agronomia) - Escola Superior de Agricultura Luiz de Queiroz Universidade de São Paulo. Piracicaba. 2001.

SAATCHI, S. et al. Persistent effeects of a severe drought on Amazonian forest canopy. Proceedings of the National Academy of Sciences of the United States os America (PNAS). Harvard University, Cambridge, MA. 2013.

SHUTTLEWORTH, W. J. GASH, J. H. C; LLOYD, C. R; MOORE, C. J; ROBERTS, J. M.; MOLION, L. C. B.; NOBRE, C. A. et al. Amazonian Evaporation. Revista Brasileira de Meteorologia. 2(1): 179 -191, 1987.

SILVA, H. J. F. ; LUCIO, P. S. ; SILVA, C. M. S. E. ; CABRAL JUNIOR, J. B. ; SANTOS, M. S. . Comparação de métodos de estimativa da Evapotranspiração de Referência com dados de estação meteorológica automática para Rio Branco-Ac. In: V Simpósio Internacional de Climatologia, 2013, Florianópolis-SC. http://www.sic2013.com/inexx/anais.

FLORIANÓPOLIS: SIC, 2013a.

SILVA, H. J. F.; CABRAL JÚNIOR, J. B.; LUCIO, P. S. Comparação de Métodos de Estimativas da Evapotranspiração para Rio Branco/AC. In: V Workshop de Mudanças Climáticas e Recursos Hídricos do Estado de Pernambuco e II Workshop Internacional sobre Mudanças
Climáticas e Biodiversidade., 2013, Recife, PE. VWMCRHPE/IIWIMB, p. 114-124. 2013b.

SOUZA, M. L. A. Comparação de métodos de estimativa da evapotranspiração de referência (ETo) em Rio Branco, Acre. 75 f. Dissertação (Mestrado em Agronomia - Produção Vegetal) UFAC, Rio Branco - Acre, 2009.

OMETTO, J. C. Bioclimatologia vegetal. São Paulo, Ed. Agronômica Ceres.1981.

WILKS, D. S. Statistical Methods in the Atmospheric Sciences. New York, USA: Academic Press, 2006.

THOM, H. C. S. Some methods of climatological analysis. WMO Technical Note 81, 1966. 53 p.

XU, M.; WEN, X.; WANG, H., ZHANG, W; DAI, $X$. et al. Effects of Climatic Factors and Ecosystem Responses on the Inter-Annual Variability of Evapotranspiration in a Coniferous Plantation in Subtropical China. PLoS ONE 9 (1), 2014. 